\title{
Neuro-Immune Networks in Gastrointestinal Disorders
}

\author{
Mohammad Khalil Zehua Zhang Matthias A. Engel \\ Department of Medicine 1, Universitätsklinikum Erlangen, Erlangen, Germany
}

\section{Keywords}

Gut-brain axis · Inflammation · Mucosal immunity · Neurons $\cdot$ Neuropeptides

\section{Abstract}

Tissue homeostasis is controlled by multilateral cell interactions. Established in autoimmune diseases of the central nervous system, growing evidence shows a fundamental role of bidirectional communication between the nervous and immune systems in various gastrointestinal disorders. Primarily the primary sensory nervous system seems to play an important role in this cross talk because of its ability for transducing inflammatory signals and to convey them to the central nervous system, which in turn responds in an efferent manner (gut-brain axis vs. brain-gut axis). Moreover, sensory neurons that play a central role in pain processing immediately respond to inflammatory stimuli through releasing a myriad of immunomodulatory neuropeptides and neurotransmitters whose receptors are expressed in different immune cell populations. Thus, a better understanding of neuro-immune networks will pave the way to novel therapeutic strategies in inflammatory as well as functional gastrointestinal disorders.

๑) 2019 S. Karger AG, Basel

\section{Introduction}

Besides fundamental physiological functions such as the transport of nutrients, digestion, and absorption, the gastrointestinal (GI) tract harbors a huge compartment of immune cells and neuronal cells. These cellular populations not only coexist, but closely interact bidirectionally on a molecular level. In addition, neuro-immune cross talk is influenced by other signals, e.g., from microbiota, connective tissue, and epithelial cells, which results in a very complex interaction. The neuronal network in the gut, also called "little brain of the gut," comprises the intrinsic enteric nervous system (iENS) (myenteric and submucosal plexus) surrounded by glial cells and the extrinsic sensory nervous system (eENS) comprising primary afferent and autonomic fibers that provide the communication between the gut and the central nervous system (CNS) [1] (for simplification, iENS and eENS will be summarized and named enteric nervous system [ENS] in this review). The ENS is the key organ to regulate gut motility, and secretion and sensory neurons within the ENS mediate painful signals to the CNS. However, peptidergic neurons have a special role with regard to its ability to impact on the mucosal immune system. The neuropeptides calcitonin gene-related peptide (CGRP) and substance P (SP) seem to be the link between neuronal activation and consecutive immune response. For instance, mice deficient of neutral endopeptidase (an enzyme responsible for the degradation of SP extracellularly) showed an aggravated trinitrobenzene sulfonic acid (TNBS) colitis [1]. This is one of the reasons why pathologic function of the ENS may have tremendous consequences in the human body and may result in both functional GI disorders such as irritable bowel syndrome (IBS) and severe inflammatory reactions such as inflammatory bowel disease (IBD) (comprising Crohn's disease [CD] and ulcerative colitis). The intriguing role of both

\section{KARGER}

(C) 2019 S. Karger AG, Basel
Matthias A. Engel, MD, PhD

Department of Medicine 1, Universitätsklinikum Erlangen

Ulmenweg 18

DE-91054 Erlangen (Germany)

E-Mail Matthias.Engel@uk-erlangen.de 
compartments, the ENS and the mucosal immune system, is reflected by the fact that patients with IBD show changes in the number of enteric neurons and glial cells including a modulated synthesis, content, and release of neurotransmitters as well as in the number of mast cells and lymphoid cells [2]. The pathophysiology of IBS and IBD is highly complex and ultimately poorly understood. It involves genetic, environmental, and psychosocial factors. Moreover, microbial alterations, impaired epithelial barrier function, and disturbances in mucosal immunology have all been implicated. Both diseases show increasing prevalence in the last decade and are a significant burden for the patient and the health economic system. Undoubtedly, increased understanding of neuro-immune networks in the GI tract will help to explore novel therapeutic strategies.

\section{Neuro-Immune Networks in IBS}

Although IBS is defined by an absence of obvious inflammation, growing evidence demonstrates that subgroups of IBS patients suffer from a chronic gut microinflammation as the driving force of their disease. IBS is defined as recurrent abdominal pain with episodes of at least 1 day per week within the last 3 months (onset 6 months) related to defecation and/or associated with a change of stool consistency and/or frequency. Having diagnosed IBS, further subclassification into IBS-C (constipation), IBS-D (diarrhea), and IBS-M (mixed type) or IBS-U (unclassified) is needed to identify an appropriate therapeutic strategy. Growing evidence indicates that the gut resident population of immune cells and its function is modified in IBS. In a subgroup of patients, microinflammation is overt and the development of postinfectious (PI)-IBS suggests that disturbed mucosal immunology forming a network with the ENS plays a pivotal role in IBS pathogenesis [3]. Human specimens from PI-IBS patients showed accumulation of T cells (PI-IBS, non-PIIBS) and mast cells (non-PI-IBS) patients $[4,5]$. Supernatants of colonic mucosal biopsies from patients with IBS showed increased levels of mast cell-derived inflammatory mediators such as histamine and serine protease, which in turn sensitize the ENS to mechanical stimuli, which results in visceral hyperalgesia in mice and excitation of nociceptive visceral sensory nerves in rat $[6,7]$. Interestingly, these increased level of mast cell mediators are evident in both IBS-C and IBS-D patients, which implicates that mast cell mediators are essential for pain signaling but not for gut motility. In addition, an increased expression of the gut-homing integrin $\beta 7$ was shown in peripheral blood lymphocytes from IBS patients [8], as a morphological correlate to the increased homing of circulating lymphocytes to the gut. In turn, function of the
ENS is altered by inflammatory mediators, which results in increased motility in patients with IBS-D. Indeed, several cytokines and chemokines such as IL- $1 \beta$, TNF- $\alpha$, IL6, IL-10, CCL3, CCL4, CCL5, and IL-12 are significantly increased in peripheral blood mononuclear cell supernatants from IBS-D patients compared to specimens from IBS-C and healthy patients [9]. Moreover, individual cytokines such as IL-1 $\beta$, TNF- $\alpha$ and IL- 6 , were shown to sensitize murine colonic afferents to mechanical stimuli via activation of the afferents expressing receptors, whereas their increased levels were found associated with pain in IBS-D patients [9]. Emphasizing the dominant role of TNF- $\alpha$ in pain processing, neutralization of TNF- $\alpha$ from peripheral blood mononuclear cell supernatants of IBS-D but not IBS-C patients by using infliximab, a TNF- $\alpha$ monoclonal antibody, attenuated significantly the mechanosensory response of murine colonic afferents [9]. In detail, TNF- $\alpha$ sensitizes murine colonic afferents through interaction between tumor necrosis factor receptor 1 and transient receptor potential ankyrin 1 (TRPA1); the latter is an ion channel with a pivotal role in pain processing [9]. The use of a selective TRPA1 antagonist prevented TNF$\alpha$-induced sensitization. However, TNF- $\alpha$-induced mechanical hypersensitivity is independent of another prominent member of the "pain ion channel family," TRPV1 (capsaicin receptor), since pharmacological blocking and genetic deletion of this channel did not alter sensitization $[9,10]$. In summary, unlike to other IBS subtypes, IBS-D seems to differ in the existence of a lowgrade inflammatory "generator" that causes disturbances in colonic motility, secretion, and neurosensory processing.

\section{Neuro-Immune Networks in IBD}

A close proximity of neuronal and immune cells in the gut lays the foundation for a close bidirectional functional interaction. On the one hand, released cytokines from immune cells modify neuronal activity and reciprocally released neuropeptides/neurotransmitters modulate immune cell function. These facts render this research area to be intensively considered in the future having a big potential to find novel treatment mechanisms [11]. Indeed, pathophysiology of IBD was shown to involve the interaction between the gut mucosal immune system and ENS [2], which involves the secretion of several mediators such as neuropeptides, serotonins (5-hydroxytryptamine, 5-HT), and acetylcholine. In fact, intestinal immune cells like macrophages, dendritic cells (DCs), lymphocytes, and mast cells express specific receptors for neuropeptides and other neuronal mediators. Neuropeptides regulate immunoglobulin production and phagocytosis $[12,13]$. Conversely, enteric neuronal cells respond 
to released cytokines from intestinal immune cells. Intestinal IL- $1 \beta$ is increased in IBD patients [14]. Furthermore, IL- $1 \beta$ and IL- 6 are capable to modulate the enteric secretomotor neurons via the suppression of norepinephrine at sympathetic synapses on the submucosal neurons in guinea pig [15] indicating the existence of a fatal neuroimmune vicious circle in inflammatory processes of the gut. Intriguingly, vice versa, destruction of the source of neuronal pro-inflammatory mediators interrupts this vicious circle. In animal models of colitis, desensitization of sensory nerves strongly attenuated colitis in mice [16]. Moreover, denervation of vagal or pelvic nerves attenuated experimental colitis as well. The fact that clonidine, nicotine, and lidocaine are seemingly effective drugs for IBD also reflects the fact that IBD is controlled by neuronal mechanisms [17].

\section{Neuropeptides and Neurotransmitters in IBD}

The supposed molecular effector molecules in neuroimmune interaction are neuropeptides and other neurotransmitters. Suggested molecular mechanisms are described in the following paragraphs.

\section{Substance $P$}

SP is an 11-amino acid neuropeptide found in both the central and peripheral nervous system. Quantitatively, the gut is an essential source of SP. SP is abundantly expressed in intrinsic (myenteric and submucosal plexuses) and extrinsic sensory neurons. SP acts on three $G$ protein-coupled receptors, neurokinin receptors 1,2 , and 3 (NK-1-3), with the highest affinity to NK-1. NK-1 is abundantly expressed in the large and small intestine not only in enteric neurons, but smooth muscle, endothelial, immune, and mucosal epithelial cells as well [2]. In both IBD patient intestinal specimens and inflamed intestinal mucosa of mice, increased SP and NK-1 receptor expression was observed [18]. However, other reports show decreased levels of mucosal SP in patients with CD, some no difference compared to healthy tissue. These controversial results may be due to different investigated regions of the gut and different stages of the disease. Namely, SP seems to act pro-inflammatorily in acute colitis [19]; however, during chronic colitis, SP was shown to enhance proliferation of intestinal epithelial cells and, thus, promote mucosal healing [20]. Thus, the putative controversy of the above results may only be the expression of a dynamic dual role of neuropeptide release in the context of intestinal inflammation. Appreciating this, the therapeutic potential of neuropeptide modulation may be related to the stages of the disease: onset of inflammation, acute exacerbation, and resolution and remission phases.

\section{CGRP and TRP Channels}

Two isoforms of CGRP are expressed ( $\alpha$-CGRP, $\beta$-CGRP), which are encoded by two different genes but are related closely to each other with predominate overlapping modes of action. $\alpha$-CGRP is essentially expressed in the central and peripheral nervous system, whereas $\beta$-CGRP is mostly expressed in the pituitary gland, immune cells, and in the gut. CGRP is highly co-expressed with some transient receptor potential (TRP) ion channels such as TRPA1 and TRPV1 (mentioned above) not only in intestinal nerve endings but also in immune cells like DCs and macrophages. Activation of TRPV1 induces release of CGRP that rather acts protective in colonic inflammation in mice $[19,21]$. In accordance, a CGRP antagonist aggravated TNBS colitis, whereas intravenous injection of CGRP attenuated TNBS colitis [1]. However, the role of TRPV1 in intestinal inflammation is still controversial since both TRPV1 activation as well as genetic deletion ameliorated dextran sulfate sodium (DSS) or TNBS colitis in rats, respectively. This putative controversy may be explained by the use of different mouse models but also by the fact that TRPV1 activation not only results in the release of CGRP, but also of many other neuropeptides/neurotransmitters, which results in yet unknown net effects on inflammation.

Other TRP channels control CGRP release as well. In a recent report, TRPM8-deficient mice showed insufficient release of neuronal CGRP into the tissue microenvironment, which led to dysregulation of DC function, which plays a fundamental role in colitis development. In these mice, CGRP treatment reversed the hyperinflammatory phenotype of TRPM8 knockout mice [22, 23]. Another study reported that systemic treatment with icilin (TRPM8 agonist) attenuated colitis severity. Moreover, icilin-treated TNBS colitic mice showed decreased levels of several inflammatory cytokines and chemokines compared to control mice. In contrast to other studies, Ramachandran et al. [24] did not show an increased colitogenic response in TRPM8-deficient mice but rather found elevated colonic level of CGRP in TRPM8-deficient mice compared to wild type (WT) mice. Furthermore, icilin-pretreated colonic tissue showed significantly decreased CGRP release by capsaicin exposure, indicating that TRPM8 activation attenuated TRPV1-stimulated CGRP release [24].

\section{Vasoactive Intestinal Peptide}

Vasoactive intestinal peptide (VIP) consists of 28 amino acids and is expressed in several tissues including the central and peripheral nervous system. The myenteric and submucosal plexus are the major source of VIP in the intestine, where VIP is responsible for chloride secretion and intestinal motility. Furthermore, VIP seems to exert anti-inflammatory effects on immune cells. An immune- 
modulatory effect of VIP is supposed to be due to its receptors expression (VPAC1 and VPAC2) in DCs, macrophages, and T cells. VIP via inhibition of NF- $\mathrm{kB}$ activation modulated the expression of pro-inflammatory cytokines such as TNF- $\alpha$, IL- 6 , and IL-12p40 and increased the expression of regulatory IL-10 in macrophages [1]. DCs seem to acquire tolerogenic properties upon VIP treatment. VIP induced activation of VPAC1, which produces low levels of costimulatory molecules CD80, CD86, and CD40 and high levels of the anti-inflammatory cytokine IL-10 upon lipopolysaccharide (LPS) stimulation [25]. Moreover, VIP enhances the survival of T helper (Th)2 but not Th1 cells via inhibition of antigen-induced apoptosis [26]. In addition, VIP attenuated the severity of TNBS colitis by suppression of Th1 and augmentation of Th2 function, indicated by decreased levels of interferon (IFN) $-\gamma$ and increased levels of IL-4 and IL-10, respectively, in the supernatants of splenic and lamina propria mononuclear cells of TNBS colitis mice treated with VIP compared to untreated mice. This renders this neuropeptide a potential candidate for the therapy of Th1-associated autoimmune disorders such as CD [27]. However, other reports did not find any beneficial effect of VIP in TNBS colitis, and VIP-deficient mice did not show aggravated colitis but rather increased resistance to DSS and TNBS colitis. Accordingly, human studies inconsistently showed up- or downregulated VIP expression in IBD specimens indicating that more research is required to address the role of VIP in the pathophysiology of IBD [1].

\section{Neuropeptide $Y$}

Neuropeptide Y (NPY) belongs to the NPY family (NPY, peptide YY, and pancreatic polypeptide); all three members consist of 36 amino acids and play a role as hormones and major modulators in the context of GI disease [28]. NPY is abundantly expressed in the CNS and implicated in nociception, appetite, energy balance, and anxiety [29]. Peripherally, it is expressed in sympathetic nerves, and submucosal as well as myenteric plexus nerves are the main source of NPY in the gut [30]. NPY is involved in gut secretion and motility [31]. Moreover, NPY serves as a paracrine and autocrine immune modulator [32]. Innate and adaptive immune cells are cell targets of NPY, where it regulates NO production, T cell differentiation, activity of natural killer cells, and antigen-presenting cells [1,32-35]. Several subtypes of Y receptor are expressed in different immune cells and species; for instance, $\mathrm{Y} 1, \mathrm{Y} 2$, and $\mathrm{Y} 5$ expression was also demonstrated in murine macrophages and rat granulocytes $[36,37]$. Therefore, the anti- or pro-inflammatory effect of NPY is dependent on Y receptor subtypes and its specific expression in certain immune cell subtypes [32]. In the context of colitis, NPY antisense oligodeoxynucleotide attenuated DSS colitis in the rat [38] and the genetic deletion of
NPY decreased the severity of DSS colitis in mice [39]. Moreover, Y1-deficient mice were protected in DSS colitis [40]. In addition, the levels of TNF- $\alpha$ and IL-12 produced by LPS-stimulated peritoneal macrophages from Y1 knockout mice were significantly decreased compared to $\mathrm{Y}^{+/+}$macrophages [40]. Interestingly, under colonic inflammatory conditions, NPY-deficient mice showed reduced levels of TNF- $\alpha$ produced by enteric neurons, and reciprocally, NPY expression was downregulated by block of TNF- $\alpha$ in vitro and in vivo [41]. Due to the diversity of its receptors in several cell populations, NPY should be intensely considered a promising novel target for IBD treatment [1].

\section{Serotonin}

Serotonin (5-HT) serves as a neurotransmitter not only in the CNS. The gut is the essential source of 5-HT in the human body and is secreted from the myenteric plexus and enterochromaffin cells. Tryptophan hydroxylase (TPH) is the responsible enzyme for $5-\mathrm{HT}$ synthesis in the intestine with two subtypes (TPH1 and TPH2). DCs isolated from DSS TPH1-deficient mice showed decreased level of released IL-12p40 compared to DCs from DSS colitic WT mice with or without LPS stimulation, indicating the role of serotonin-regulated DC activation in the context of colitis [42]. Accordingly, genetic deletion of 5-HT7 receptor attenuated dinitrobenzene sulfonic acid and DSS colitis, and 5-HT7 receptor antagonism attenuated DSS colitis as well [43]. In addition, deletion of the serotonin reuptake transporter in IL-10-deficient mice that develop spontaneous colitis exacerbated colonic inflammation, indicating once more the pro-inflammatory role of 5-HT in the gut [44]. However, 5-HT receptors that are expressed in intestinal DCs are also involved in CNS and ENS function. Therefore, modulation of 5-HT signaling may induce serious side effects such as motility and behavioral disturbance [1]. In summary, selective blockage of TPH-1 may provide a new therapeutic tool for IBD patients; however, rigorous clinical studies regarding its potential to induce psychiatric disorders such as depression have to be conducted [1].

\section{TRP Channels - Linking Neurons to Immune Cells}

Many studies have described the role of TRP channels in modification of several models of colitis via neurogenic inflammation (modulation of neuropeptide release). We have previously reported that TRPA1-induced SP release from extrinsic afferent neurons was essential for the development and maintenance of experimental colitis. Pharmacologic blocking of TRPA1 ameliorated chronic colitis via inhibition of neuropeptide release [19]. However, it is increasingly recognized that TRP ion channels 
are also functionally expressed in non-neuronal cells, which results in a complex biological response upon TRP activation. TRPA1 and TRPV1 expression in healthy and inflamed colonic tissues of human and mice was recently reported in epithelial cells and macrophages [45]. TRPA1-deficient mice showed increased severity of DSS colitis compared to WTs mirrored by increased levels of proinflammatory neuropeptide mediators such as SP and neurokinins $\mathrm{A}$ and $\mathrm{B}$ in the distal colon homogenates. Genetic deletion of TRPA1 in CD4 ${ }^{+} \mathrm{T}$ cells caused intestinal inflammation via induction of transcription factor T-bet, which consequently increased production of the pro-inflammatory cytokines IFN- $\gamma$ and IL-2 [46], which enhanced the differentiation of T cells into Th1 effector cells. Furthermore, the lack of TRPV 1 in CD $4^{+} \mathrm{T}$ cells attenuated calcium influx upon $\mathrm{T}$-cell receptor stimulation, leading to inactivation of the transcription factor nuclear factor of activated $\mathrm{T}$ cells and NF- $\mathrm{B}$ [47]. In vivo, IL-10 knockout mice showed increased severity of piroxicam colitis compared to IL-10/TRPV1 double knockout mice. Furthermore, TRPV1 antagonist (SB366791)-treated IL10-deficient mice showed a lower grade of colitis and decreased levels of T-cell-derived inflammatory cytokines compared to control IL-10 mice. Furthermore, adoptive transfer of Trpv1 $1^{-/-}$naive $\mathrm{CD} 4^{+} \mathrm{T}$ cells in $\mathrm{Rag} 1^{-/-}$mice showed an attenuated colitis, indicated by decreased loss of body weight, lower disease activity index, and decreased levels of several pro-inflammatory cytokines such as IFN- $\gamma$, IL-17A, and TNF compared to adoptive transfer of WT naive CD4 ${ }^{+}$T cells [47]. Other TRP channels were recently involved in IBD pathogenesis via different mechanisms. Upregulation of TRPV4 mRNA was shown in colonic intestinal epithelial cells from DSS colitic mice. TRPV4 activation led to cytokine and chemokine release such as IL-8, IP-10, MIG, and MCP-1, which reflected a pivotal role of TRPV4 in activating pro-inflammatory signaling pathways in inducing the recruitment of immune cells such as macrophages. In addition, intrarectal administration of $4 \alpha$-PDD (TRPV4 agonist) induced acute and chronic colonic inflammation in mice [48]. We could recently show that mice that were reconstituted with TRPM8-deficient macrophages exhibited aggravated colitis, reflecting a potential role of constitutive TRPM8 expression in macrophages in the context of colitis [49]. However, TRPM8 activation also has important neuronal effects. The TRPM8 agonists menthol (100 mM) and icilin (33 mM) abolished mechanically induced (90 mm Hg) neuropeptide (CGRP) release in the healthy colon, whereas both agonists were insufficient to show any effect in an inflamed colon (2\% DSS colitis) where neuropeptide release was per se strongly attenuated. Colitis-induced depletion/desensitization of peptidergic nerve endings with consecutive reduced/altered neuropeptide release may be a central pathogenetic step in colitis maintenance [49].

\section{The Microbial Gut-Brain Axis}

Gut-brain and brain-gut bidirectional interaction is fundamental to metabolic survival. Intestinal microbes maintain gut homeostasis by being implicated in digestion, by inducing immune responses, and by interaction with other organs such as the brain. About $10^{13}$ microbes involving 1,000 species are located in the colon [50]. Intriguingly, recent reports indicate that microbiota, among other things such as metabolism and emotional behavior, also regulate immune response and neuronal networks $[51,52]$. In experiments with germ-free mice, one could show that the number of ENS cells was strongly decreased compared to their controls, which resulted in decelerated gut motility [53]. Moreover, mucosal enteric glial cells were dysregulated with attenuated influx into the intestinal mucosa in antibiotics-treated mice compared to control mice [54]. This state could be reversed by reconstitution with microbiota, which reflects the fundamental role of microbiota in the neuronal intestinal composition [54]. Gut microbiota not only act directly on cells within their microenvironment (neuronal, epithelial, and immune cells) but rather on distant organs as well. Several studies reported that a significant part of metabolites in mammalian blood are derived from the community of intestinal microbiota [55]. Via released molecules such as microbeassociated molecular patterns (MAMPs) and other microbial metabolites, microbiota communicate with other organs [56]. LPS, which belongs to the MAMP family, modulates distant organs via Toll-like receptor 4 (TLR4) action via different mechanisms [56]. Noteworthily, TLR4 is expressed at several levels of the gut-brain axis such as in intestinal epithelial cells, enteric neurons, primary afferent neurons, and several cell populations in the brain (microglia, neurons, astrocytes) [57-60]. LPS-stimulated cytokine production in intestinal immune cells sensitizes vagal and spinal afferent neurons (DRGs) and thus alter brain function [61], a phenomenon described shortly as the microbial gut-brain axis [62]. LPS in the circulating blood stream was shown to alter brain function even directly [63]. Various bacterial factors including LPS were shown to stimulate the release of pro-inflammatory cytokines in the brain, which led to increased risk of neuroinflammation and manifestation of psychiatric disorders $[64,65]$. For instance, LPS was implicated in the pathophysiology of depression and chronic fatigue syndrome $[66,67]$. It was hypothesized that LPS released by intestinal microbiota induced increased blood levels of IgA and IgM, which may have an impact in these disorders [66, 67]. Moreover, recent reports suggest an initial intestinal origin of Parkinson's disease (PD). PD patients showed gut dysbiosis and accumulation of $\alpha$-synuclein in enteric neurons several years before motor symptoms developed [68]. An experimental approach in rats where $\alpha$-synuclein
56

Visc Med 2019;35:52-60 DOI: $10.1159 / 000496838$
Khalil/Zhang/Engel 


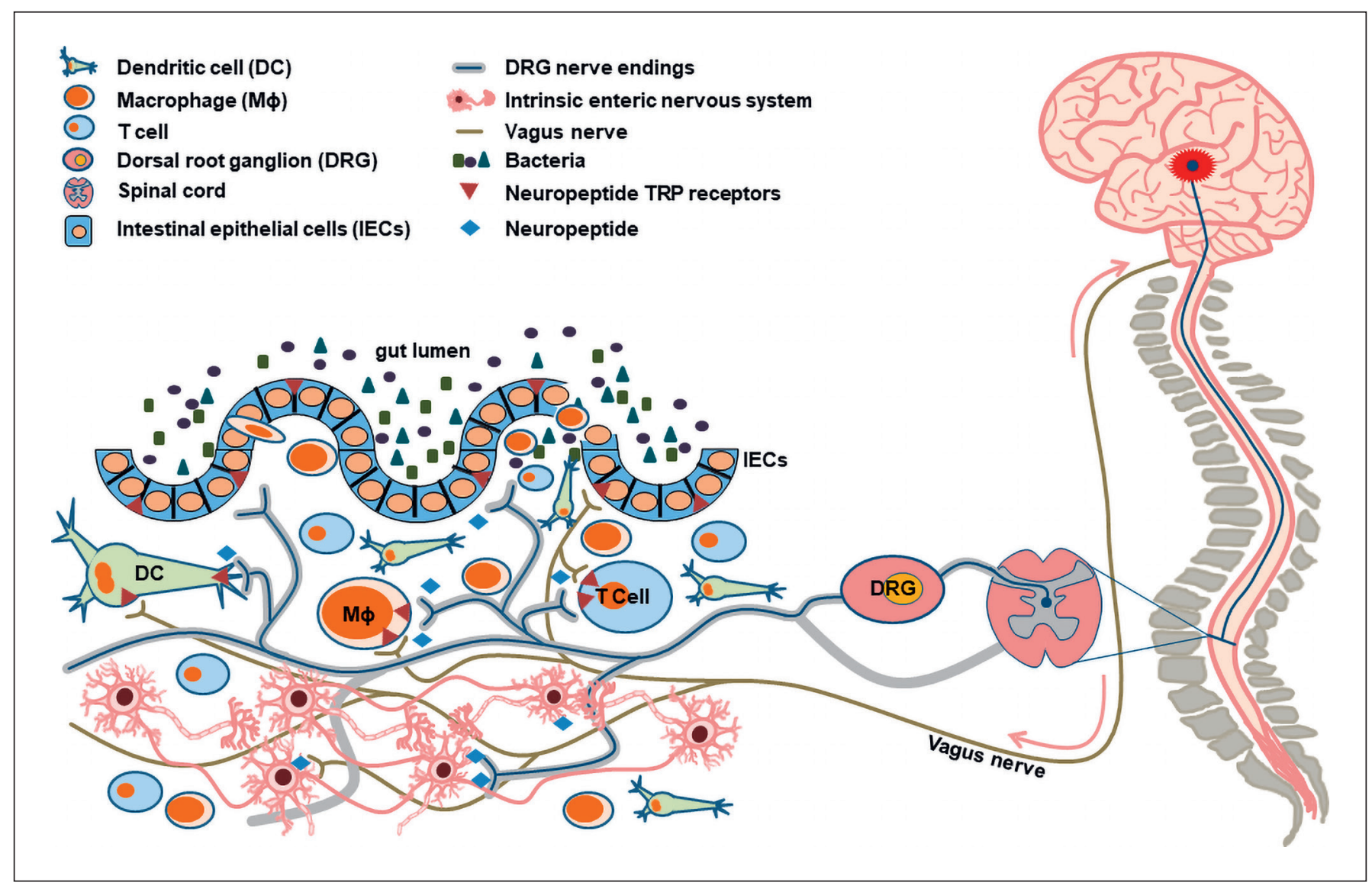

Fig. 1. Neuro-immune networks in the gut regulate colonic homeostasis in the steady state and under inflammatory conditions. The multilateral interaction between neurons, immune cells, and epithelial cells is fundamental to maintain the immunological balance in the gut. Immune cells express several neuropeptide receptors such as CGRP, SP, and NPY receptors and thus are direct target cells of neuronal action. At the same time, the three cell compartments express several TRP ion channels, which results in a complex interplay. Vice versa, under colonic inflammatory conditions, immune cells release several proinflammatory mediators such as IL-1 $\beta$, which in turn activate/sensitize peptidergic sensory neurons and induce pro-inflammatory SP release [84]. Moreover, neuronal and epithelial IL- 8 expression is controlled by IL- $1 \beta$ via MAPK signaling [85]. In addition, probiotic microorganisms control gut immune responses through their ability to induce proliferation of T regulatory cells and dendritic cells [80, 81]. Moreover, bidirectional communication between the gut and the brain, termed gut-brain axis or brain-gut axis, respectively, modulate these circuits on a superior level $[69,70]$. was injected into the gut wall and translocated via vagus nerve fibers into the dorsal motor nucleus of the vagal nerve suggested that this spreading mechanism could also apply in humans [69]. An increase of glial cell markers such as GFAP, SOX10, and pro-inflammatory cytokine genes in the gut of PD patients suggested the relationship between PD development and intestinal inflammation/ glial dysregulation and may therefore become a potential early marker of PD in humans [70].

Several animal experimental models indicate the implication of gut bacteria in IBD pathophysiology $[71,72]$. Antibiotic pretreated mice were shown to attenuate intestinal chemically induced inflammation $[73,74]$. In patients with IBD, a Helicobacter, Campylobacter, and Enterococcus species-dominant microbial profile was reported $[75,76]$. On the other hand, an aberrated microbial profile can be improved by the application of probiotic microorganisms [77]. These organisms directly attenuate toxic microbial metabolic activities and modulate intestinal immune response and thus control inflammation [77, 78]. Probiotic microorganisms were shown to induce an anti-inflammatory/regulatory response in epithelial cells and DCs and could induce proliferation of regulatory $\mathrm{T}$ cells in the gut mucosa [79-81]. The probiotic cocktail VSL\#3 was shown effective in TNBS colitis [82]. The proposed cellular mechanism was the induction of an anti-inflammatory $\mathrm{T}$-cell phenotype, which was mirrored by an increased number of TGF- $\beta$-expressing $\mathrm{T}$ cells and IL-10 expression. Adoptive transfer of lamina propria mononuclear cells from VSL\#3-treated mice into WT recipient mice inhibited colitis; however, when TGF$\beta$-bearing $\mathrm{CD}^{+} \mathrm{T}$ cells were depleted before transfer, this beneficial effect was abolished, emphasizing a crucial link between probiotics and $\mathrm{CD} 4^{+} \mathrm{T}$ cells [82]. In accordance, 
Bifidobacteria infantis-treated mice showed induction of Treg cells and protective effects against inflammation during infection with Salmonella typhimurium [83]. In addition, adoptive transfer of $\mathrm{CD} 4^{+} \mathrm{CD} 25^{+} \mathrm{T}$ cells from B. infantis-treated mice reduced in vivo NF- $\kappa \mathrm{B}$ activation followed by LPS injection in recipient mice [83].

\section{Conclusion}

Neuro-immune networks are fundamental in the regulation of a sensitive and highly complex cellular cross talk in gut tissue. Small bidirectional circuits between neurons and immune cells are controlled by neuropeptide/cytokine interactions in close proximity, but these again are regulated by bigger circuits, namely, the gutbrain or brain-gut axis, which are again modulated by environmental or genetic factors (Fig. 1). Elucidating mechanisms of neuro-immune interaction is crucial for the development of novel therapeutic strategies against GI disorders and cannot be neglected given the fact that the gut harbors the highest number and density of neurons besides the CNS and the growing evidence for important functional neuro-immune networks.

\section{Statement of Ethics}

The authors have no ethical conflicts to disclose.

\section{Disclosure Statement}

The authors have no conflicts of interest to disclose.

\section{Funding Sources}

M.K. and M.A.E. received support from the Deutsche Forschungsgemeinschaft (DFG-SFB/TRR 241[B05]).

\section{Author Contributions}

M.K.: conception and writing of the manuscript; Z.Z.: writing of the manuscript, illustration; M.A.E.: conception and writing of the manuscript, critical revision for important intellectual content, and final approval of the version to be published.

\section{References}

1 Di Giovangiulio M, Verheijden S, Bosmans G, Stakenborg N, Boeckxstaens GE, Matteoli G. The Neuromodulation of the Intestinal Immune System and Its Relevance in Inflammatory Bowel Disease. Front Immunol. 2015 Nov; 6:590.

2 Margolis KG, Gershon MD. Neuropeptides and inflammatory bowel disease. Curr Opin Gastroenterol. 2009 Nov;25(6):503-11.

3 Spiller R, Garsed K. Postinfectious irritable bowel syndrome. Gastroenterology. 2009 May;136(6):1979-88.

4 Spiller RC, Jenkins D, Thornley JP, Hebden $\mathrm{JM}$, Wright $\mathrm{T}$, Skinner $\mathrm{M}$, et al. Increased rectal mucosal enteroendocrine cells, T lymphocytes, and increased gut permeability following acute Campylobacter enteritis and in post-dysenteric irritable bowel syndrome. Gut. 2000 Dec;47(6):804-11.

5 Dunlop SP, Jenkins D, Spiller RC. Distinctive clinical, psychological, and histological features of postinfective irritable bowel syndrome. Am J Gastroenterol. 2003 Jul;98(7): 1578-83.

6 Cenac N, Andrews CN, Holzhausen M, Chapman K, Cottrell G, Andrade-Gordon P, et al. Role for protease activity in visceral pain in irritable bowel syndrome. J Clin Invest. 2007 Mar;117(3):636-47.

7 Barbara G, Wang B, Stanghellini V, de Giorgio R, Cremon C, Di Nardo G, et al. Mast celldependent excitation of visceral-nociceptive sensory neurons in irritable bowel syndrome. Gastroenterology. 2007 Jan;132(1):26-37.
8 Ohman L, Isaksson S, Lundgren A, Simrén M, Sjövall H. A controlled study of colonic immune activity and $\beta 7+$ blood $\mathrm{T}$ lymphocytes in patients with irritable bowel syndrome. Clin Gastroenterol Hepatol. 2005 Oct;3(10): 980-6.

9 Hughes PA, Harrington AM, Castro J, Liebregts T, Adam B, Grasby DJ, et al. Sensory neuro-immune interactions differ between irritable bowel syndrome subtypes. Gut. 2013 Oct;62(10):1456-65.

10 Jin X, Gereau RW 4th. Acute p38-mediated modulation of tetrodotoxin-resistant sodium channels in mouse sensory neurons by tumor necrosis factor-alpha. J Neurosci. 2006 Jan; 26(1):246-55

11 Yoo BB, Mazmanian SK. The Enteric Network: Interactions between the Immune and Nervous Systems of the Gut. Immunity. 2017 Jun;46(6):910-26.

12 Ferreira R, Santos T, Viegas M, Cortes L, Bernardino L, Vieira OV, et al. Neuropeptide Y inhibits interleukin- $1 \beta$-induced phagocytosis by microglial cells. J Neuroinflammation. 2011 Dec;8(1): 169

13 Friedman EM, Irwin MR, Nonogaki K. Neuropeptide $\mathrm{Y}$ inhibits in vivo specific antibody production in rats. Brain Behav Immun. 1995 Sep;9(3):182-9.

14 Ligumsky M, Simon PL, Karmeli F, Rachmilewitz D. Role of interleukin 1 in inflammatory bowel disease-enhanced production during active disease. Gut. 1990 Jun;31(6): $686-9$.
15 Xia Y, Hu HZ, Liu S, Ren J, Zafirov DH, Wood JD. IL-1beta and IL-6 excite neurons and suppress nicotinic and noradrenergic neurotransmission in guinea pig enteric nervous system. J Clin Invest. 1999 May;103(9):1309_ 16.

16 Kistner K, Siklosi N, Babes A, Khalil M, Selescu T, Zimmermann K, et al. Systemic desensitization through TRPA1 channels by capsazepine and mustard oil - a novel strategy against inflammation and pain. Sci Rep. 2016 Jun;6(1):28621.

17 Collins SM. The immunomodulation of enteric neuromuscular function: implications for motility and inflammatory disorders. Gastroenterology. 1996 Dec;111(6):1683-99.

18 Sideri A, Bakirtzi K, Shih DQ, Koon HW, Fleshner P, Arsenescu R, et al. Substance P mediates pro-inflammatory cytokine release form mesenteric adipocytes in Inflammatory Bowel Disease patients. Cell Mol Gastroenterol Hepatol. 2015 Jul;1(4):420-32.

19 Engel MA, Leffler A, Niedermirtl F, Babes A, Zimmermann K, Filipović MR, et al. TRPA1 and substance $\mathrm{P}$ mediate colitis in mice. Gastroenterology. 2011 Oct;141(4):1346-58.

20 Koon HW, Zhao D, Na X, Moyer MP, Pothoulakis C. Metalloproteinases and transforming growth factor-alpha mediate substance P-induced mitogen-activated protein kinase activation and proliferation in human colonocytes. J Biol Chem. 2004 Oct;279(44):4551927. 
21 Engel MA, Khalil M, Mueller-Tribbensee SM, Becker C, Neuhuber WL, Neurath MF, et al. The proximodistal aggravation of colitis depends on substance P released from TRPV1expressing sensory neurons. J Gastroenterol. 2012 Mar;47(3):256-65.

22 Abe K, Nguyen KP, Fine SD, Mo JH, Shen C, Shenouda $S$, et al. Conventional dendritic cells regulate the outcome of colonic inflammation independently of T cells. Proc Natl Acad Sci USA. 2007 Oct;104(43):17022-7.

23 de Jong PR, Takahashi N, Peiris M, Bertin S, Lee J, Gareau MG, et al. TRPM8 on mucosal sensory nerves regulates colitogenic responses by innate immune cells via CGRP. Mucosal Immunol. 2015 May;8(3):491-504.

24 Ramachandran R, Hyun E, Zhao L, Lapointe TK, Chapman K, Hirota CL, et al. TRPM8 activation attenuates inflammatory responses in mouse models of colitis. Proc Natl Acad Sci USA. 2013 Apr;110(18):7476-81.

25 Delgado M, Gonzalez-Rey E, Ganea D. The neuropeptide vasoactive intestinal peptide generates tolerogenic dendritic cells. J Immunol. 2005 Dec;175(11):7311-24.

26 Delgado M, Leceta J, Ganea D. Vasoactive intestinal peptide and pituitary adenylate cyclase-activating polypeptide promote in vivo generation of memory Th2 cells. FASEB J. 2002 Nov;16(13):1844-6.

27 Abad C, Martinez C, Juarranz MG, Arranz A, Leceta J, Delgado M, et al. Therapeutic effects of vasoactive intestinal peptide in the trinitrobenzene sulfonic acid mice model of Crohn's disease. Gastroenterology. 2003 Apr;124(4): 961-71.

28 Vona-Davis LC, McFadden DW. NPY family of hormones: clinical relevance and potential use in gastrointestinal disease. Curr Top Med Chem. 2007;7(17):1710-20.

29 Bannon AW, Seda J, Carmouche M, Francis JM, Norman MH, Karbon B, et al. Behavioral characterization of neuropeptide Y knockout mice. Brain Res. 2000 Jun;868(1):79-87.

30 Holzer P, Reichmann F, Farzi A. Neuropeptide $Y$, peptide $Y Y$ and pancreatic polypeptide in the gut-brain axis. Neuropeptides. 2012 Dec;46(6):261-74.

31 Sheikh SP. Neuropeptide Y and peptide YY: major modulators of gastrointestinal blood flow and function. Am J Physiol. 1991 Nov; 261(5 Pt 1):G701-15.

32 Farzi A, Reichmann F, Holzer P. The homeostatic role of neuropeptide $\mathrm{Y}$ in immune function and its impact on mood and behaviour. Acta Physiol (Oxf). 2015 Mar;213(3):603-27.

33 Saurer TB, Ijames SG, Lysle DT. Neuropeptide Y Y1 receptors mediate morphine-induced reductions of natural killer cell activity. J Neuroimmunol. 2006 Aug;177(1-2):18-26.

34 Medina S, Del Río M, Hernanz A, De la Fuente M. Age-related changes in the neuropeptide $Y$ effects on murine lymphoproliferation and interleukin-2 production. Peptides. 2000 Sep;21(9):1403-9.

35 Bedoui S, Kawamura N, Straub RH, Pabst R, Yamamura T, von Hörsten S. Relevance of neuropeptide $\mathrm{Y}$ for the neuroimmune crosstalk. J Neuroimmunol. 2003 Jan;134(1-2):111 .
36 Mitić K, Stanojević S, Kuštrimović N, Vujić V, Dimitrijević M. Neuropeptide Y modulates functions of inflammatory cells in the rat: distinct role for Y1, Y2 and Y5 receptors. Peptides. 2011 Aug;32(8):1626-33.

37 Singer K, Morris DL, Oatmen KE, Wang T, DelProposto J, Mergian T, et al. Neuropeptide $\mathrm{Y}$ is produced by adipose tissue macrophages and regulates obesity-induced inflammation. PLoS One. 2013;8(3):e57929.

38 Pang XH, Li TK, Xie Q, He FQ, Cui DJ, Chen YQ, et al. Amelioration of dextran sulfate sodium-induced colitis by neuropeptide $\mathrm{Y}$ antisense oligodeoxynucleotide. Int J Colorectal Dis. 2010 Sep;25(9):1047-53.

39 Chandrasekharan B, Bala V, Kolachala VL, Vijay-Kumar M, Jones D, Gewirtz AT, et al. Targeted deletion of neuropeptide Y (NPY) modulates experimental colitis. PLoS One. 2008 Oct;3(10):e3304.

40 Wheway J, Mackay CR, Newton RA, Sainsbury A, Boey D, Herzog H, et al. A fundamental bimodal role for neuropeptide Y1 receptor in the immune system. J Exp Med. 2005 Dec; 202(11):1527-38.

41 Chandrasekharan B, Jeppsson S, Pienkowski S, Belsham DD, Sitaraman SV, Merlin D, et al. Tumor necrosis factor-neuropeptide Y cross talk regulates inflammation, epithelial barrier functions, and colonic motility. Inflamm Bowel Dis. 2013 Nov; 19(12):2535-46.

42 Li N, Ghia JE, Wang H, McClemens J, Cote F, Suehiro Y, et al. Serotonin activates dendritic cell function in the context of gut inflammation. Am J Pathol. 2011 Feb;178(2):662-71.

43 Kim JJ, Bridle BW, Ghia JE, Wang H, Syed SN, Manocha MM, et al. Targeted inhibition of serotonin type 7 (5-HT7) receptor function modulates immune responses and reduces the severity of intestinal inflammation. J Immunol. 2013 May;190(9):4795-804.

44 Haub S, Ritze Y, Bergheim I, Pabst O, Gershon MD, Bischoff SC: Enhancement of intestinal inflammation in mice lacking interleukin 10 by deletion of the serotonin reuptake transporter. Neurogastroenterol Motil. 2010 Jul;22(7):826-34, e229.

45 Kun J, Szitter I, Kemény A, Perkecz A, Kereskai L, Pohóczky K, et al. Upregulation of the transient receptor potential ankyrin 1 ion channel in the inflamed human and mouse colon and its protective roles. PLoS One. 2014 Sep;9(9):e108164.

46 Bertin S, Aoki-Nonaka Y, Lee J, de Jong PR, Kim P, Han T, et al. The TRPA1 ion channel is expressed in CD4+ T cells and restrains Tcell-mediated colitis through inhibition of TRPV1. Gut. 2017 Sep;66(9):1584-96.

47 Bertin S, Aoki-Nonaka Y, de Jong PR, Nohara LL, Xu H, Stanwood SR, et al. The ion channel TRPV1 regulates the activation and proinflammatory properties of CD4区 $\mathrm{T}$ cells. Nat Immunol. 2014 Nov; 15(11):1055-63.

48 D'Aldebert E, Cenac N, Rousset P, Martin L, Rolland C, Chapman K, et al. Transient receptor potential vanilloid 4 activated inflammatory signals by intestinal epithelial cells and colitis in mice. Gastroenterology. 2011 Jan; 140(1):275-85.
49 Khalil M, Babes A, Lakra R, Försch S, Reeh PW, Wirtz S, et al. Transient receptor potential melastatin 8 ion channel in macrophages modulates colitis through a balance-shift in TNF-alpha and interleukin-10 production. Mucosal Immunol. 2016 Nov;9(6):1500-13.

50 Cryan JF, Dinan TG. Mind-altering microorganisms: the impact of the gut microbiota on brain and behaviour. Nat Rev Neurosci. 2012 Oct;13(10):701-12.

51 Tremaroli V, Bäckhed F. Functional interactions between the gut microbiota and host metabolism. Nature. 2012 Sep;489(7415): 242-9.

52 Lee WJ, Hase K. Gut microbiota-generated metabolites in animal health and disease. Nat Chem Biol. 2014 Jun;10(6):416-24.

53 Obata Y, Pachnis V. The Effect of Microbiota and the Immune System on the Development and Organization of the Enteric Nervous System. Gastroenterology. 2016 Nov;151(5): 836-44.

54 Kabouridis PS, Lasrado R, McCallum S, Chng $\mathrm{SH}$, Snippert HJ, Clevers H, et al. Microbiota controls the homeostasis of glial cells in the gut lamina propria. Neuron. 2015 Jan;85(2): 289-95.

55 Wikoff WR, Anfora AT, Liu J, Schultz PG, Lesley SA, Peters EC, et al. Metabolomics analysis reveals large effects of gut microflora on mammalian blood metabolites. Proc Natl Acad Sci USA. 2009 Mar;106(10):3698-703.

56 Holzer P, Farzi A, Hassan AM, Zenz G, Jačan A, Reichmann F. Visceral Inflammation and Immune Activation Stress the Brain. Front Immunol. 2017 Nov; 8:1613.

57 Abreu MT. Toll-like receptor signalling in the intestinal epithelium: how bacterial recognition shapes intestinal function. Nat Rev Immunol. 2010 Feb;10(2):131-44.

58 Barajon I, Serrao G, Arnaboldi F, Opizzi E, Ripamonti G, Balsari A, et al. Toll-like receptors 3,4 , and 7 are expressed in the enteric nervous system and dorsal root ganglia. J Histochem Cytochem. 2009 Nov;57(11):101323.

59 van Noort JM, Bsibsi M: Toll-like receptors in the CNS: implications for neurodegeneration and repair. Prog Brain Res. 2009;175:139-48.

60 Tse KH, Chow KB, Leung WK, Wong YH, Wise $H$. Primary sensory neurons regulate Toll-like receptor-4-dependent activity of glial cells in dorsal root ganglia. Neuroscience. 2014 Oct;279:10-22.

61 Holzer P, Farzi A. Neuropeptides and the microbiota-gut-brain axis. Adv Exp Med Biol. 2014;817:195-219.

62 Montiel-Castro AJ, González-Cervantes RM, Bravo-Ruiseco G, Pacheco-López G. The microbiota-gut-brain axis: neurobehavioral correlates, health and sociality. Front Integr Nuerosci. 2013 Oct; 7:70.

63 Pardon MC. Lipopolysaccharide hyporesponsiveness: protective or damaging response to the brain? Rom J Morphol Embryol. 2015;56(3):903-13.

64 Czerniawski J, Guzowski JF. Acute neuroinflammation impairs context discrimination memory and disrupts pattern separation processes in hippocampus. J Neurosci. 2014 Sep; 34(37):12470-80. 
65 Rudzki L, Szulc A. "Immune Gate” of Psychopathology-The Role of Gut Derived Immune Activation in Major Psychiatric Disorders. Front Psychiatry. 2018 May; 9:205.

66 Maes M, Kubera M, Leunis JC, Berk M. Increased IgA and IgM responses against gut commensals in chronic depression: further evidence for increased bacterial translocation or leaky gut. J Affect Disord. 2012 Dec;141(1): 55-62.

67 Maes M, Twisk FN, Kubera M, Ringel K, Leunis JC, Geffard M. Increased IgA responses to the LPS of commensal bacteria is associated with inflammation and activation of cell-mediated immunity in chronic fatigue syndrome. J Affect Disord. 2012 Feb;136(3):90917.

68 Shannon KM, Keshavarzian A, Dodiya HB, Jakate S, Kordower JH. Is alpha-synuclein in the colon a biomarker for premotor Parkinson's disease? Evidence from 3 cases. Mov Disord. 2012 May;27(6):716-9.

69 Holmqvist S, Chutna O, Bousset L, AldrinKirk P, Li W, Björklund T, et al. Direct evidence of Parkinson pathology spread from the gastrointestinal tract to the brain in rats. Acta Neuropathol. 2014 Dec;128(6):805-20.

70 Devos D, Lebouvier T, Lardeux B, Biraud M, Rouaud T, Pouclet $\mathrm{H}$, et al. Colonic inflammation in Parkinson's disease. Neurobiol Dis. 2013 Feb;50:42-8.

71 Sartor RB, Wu GD: Roles for Intestinal Bacteria, Viruses, and Fungi in Pathogenesis of Inflammatory Bowel Diseases and Therapeutic Approaches. Gastroenterology. 2017 Feb; 152(2):327-339.e4.
72 Minamoto Y, Otoni CC, Steelman SM, Büyükleblebici O, Steiner JM, Jergens AE, et al. Alteration of the fecal microbiota and serum metabolite profiles in dogs with idiopathic inflammatory bowel disease. Gut Microbes. 2015;6(1):33-47.

73 Hernández-Chirlaque C, Aranda CJ, Ocón B, Capitán-Cañadas F, Ortega-González M, Carrero JJ, et al. Germ-free and Antibiotictreated Mice are Highly Susceptible to Epithelial Injury in DSS Colitis. J Crohn's Colitis. 2016 Nov;10(11):1324-35.

74 Ward NL, Phillips CD, Nguyen DD, Shanmugam NK, Song Y, Hodin R, et al. Antibiotic Treatment Induces Long-lasting Changes in the Fecal Microbiota that Protect Against Colitis. Inflamm Bowel Dis. 2016 Oct;22(10): 2328-40.

75 Castaño-Rodríguez N, Kaakoush NO, Lee WS, Mitchell HM. Dual role of Helicobacter and Campylobacter species in IBD: a systematic review and meta-analysis. Gut. 2017 Feb; 66(2):235-49.

76 Zhou Y, Chen H, He H, Du Y, Hu J, Li Y, et al. Increased Enterococcus faecalis infection is associated with clinically active Crohn disease. Medicine (Baltimore). 2016 Sep; 95(39):e5019.

77 Vemuri R, Gundamaraju R, Eri R. Role of Lactic Acid Probiotic Bacteria in IBD. Curr Pharm Des. 2017;23(16):2352-5.

78 Round JL, Mazmanian SK. The gut microbiota shapes intestinal immune responses during health and disease. Nat Rev Immunol. 2009 May;9(5):313-23.

79 Wan LY, Chen ZJ, Shah NP, El-Nezami H. Modulation of Intestinal Epithelial Defense Responses by Probiotic Bacteria. Crit Rev Food Sci Nutr. 2016 Dec;56(16):2628-41.
80 You J, Dong H, Mann ER, Knight SC, Yaqoob P. Probiotic modulation of dendritic cell function is influenced by ageing. Immunobiology. 2014 Feb;219(2):138-48.

81 Rossi G, Pengo G, Caldin M, Palumbo Piccionello A, Steiner JM, Cohen ND, et al. Comparison of microbiological, histological, and immunomodulatory parameters in response to treatment with either combination therapy with prednisone and metronidazole or probiotic VSL\#3 strains in dogs with idiopathic inflammatory bowel disease. PLoS One. 2014 Apr;9(4):e94699.

82 Di Giacinto C, Marinaro M, Sanchez M, Strober W, Boirivant M. Probiotics ameliorate recurrent Th1-mediated murine colitis by inducing IL-10 and IL-10-dependent TGF$\beta$-bearing regulatory cells. J Immunol. 2005 Mar;174(6):3237-46.

83 O'Mahony C, Scully P, O’Mahony D, Murphy S, O'Brien F, Lyons A, et al. Commensal-induced regulatory $\mathrm{T}$ cells mediate protection against pathogen-stimulated NF-kappaB activation. PLoS Pathog. 2008 Aug; 4(8): e1000112.

84 Perretti M, Ahluwalia A, Flower RJ, Manzini S. Endogenous tachykinins play a role in IL1 -induced neutrophil accumulation: involvement of NK-1 receptors. Immunology. 1993 Sep;80(1):73-7.

85 Tixier E, Lalanne F, Just I, Galmiche JP, Neunlist M. Human mucosa/submucosa interactions during intestinal inflammation: involvement of the enteric nervous system in interleukin-8 secretion. Cell Microbiol. 2005 Dec;7(12):1798-810. 